\title{
Opposite effects of short- and long-term fatty acid infusion on insulin secretion in healthy subjects
}

\author{
G. Paolisso, A. Gambardella, L. Amato, R. Tortoriello, A. D'Amore, M. Varricchio, F. D'Onofrio \\ Department of Geriatric Medicine and Metabolic Diseases, II University of Naples, Naples, Italy
}

Summary Our study investigates short- and longterm effects of infusion of non-esterified fatty acids (NEFA) on insulin secretion in healthy subjects. Twelve healthy individuals underwent a $24-\mathrm{h}$ Intralipid (10\% triglyceride emulsion) infusion at a rate of $0.4 \mathrm{ml} / \mathrm{min}$ with a simultaneous infusion of heparin (a bolus of $200 \mathrm{U}$ followed by $0.2 \mathrm{U} / \mathrm{min}$ per $\mathrm{kg}$ body weight). After an overnight fast (baseline), at 6 and at $24 \mathrm{~h}$ of Intralipid infusion and $24 \mathrm{~h}$ after Intralipid discontinuation (recovery test), all subjects underwent an intravenous glucose tolerance test (ivGTT) (25 g of glucose/min). Intralipid infusion caused a threefold rise in plasma NEFA concentrations with no difference between the 6- and the 24-h concentrations. Compared to baseline acute insulin response (AIR) (AIR $=63 \pm 8 \mathrm{mU} / \mathrm{l})$, short-term (6h) Intralipid infusion was associated with a significant increase in AIR $(86 \pm 12 \mathrm{mU} / 1 p<0.01)$; in contrast, long-term (24-h) Intralipid delivery was associated with inhibition of AIR $(31 \pm 5 \mathrm{mU} / \mathrm{l})$ compared to baseline $(p<0.001)$ and to the $6-\mathrm{h}(p<0.03)$ triglycer- ide emulsion infusion. Intralipid infusion was associated with a progressive and significant decline in respiratory quotient (RQ). A positive correlation between changes in fasting plasma NEFA concentrations and AIR at the 6-h infusion $(r=0.89 p<0.001)$ was found. In contrast, at the end of the Intralipid infusion period, changes in plasma NEFA concentrations and AIR were negatively correlated $(r=-0.87$ $p<0.001$ ). The recovery test showed that fasting plasma NEFA concentrations, RQ and AIR had returned to baseline values. In the control study $(n=8) 0.9 \%$ $\mathrm{NaCl}$ infusion did not mimick the effect of Intralipid. In conclusion, our study demonstrates that shortand long-term exposures of beta cells to high plasma NEFA concentrations have opposite effects on glucose-induced insulin secretion. [Diabetologia (1995) 38: 1295-1299]

Key words Non-esterified fatty acids, plasma insulin, acute insulin response, respiratory quotient.
In vitro [1] and in vivo [2] studies performed in the 1960 s and 1970 s showed that non-esterified fatty acids (NEFA) stimulate insulin secretion. The effect of NEFA on beta-cell secretion is normally moderate, however, indicating that these acids do not play a ma-

Received: 11 February 1995 and in revised form: 11 April 1995

Corresponding author: Dr. G. Paolisso, Department of Geriatric Medicine and Metabolic Diseases, Servizio di Astanteria Medica - I Policlinico, Piazza Miraglia, 2, I-80138 Napoli, Italy Abbreviations: NEFA, Non-esterified fatty acids; ivGTT, intravenous glucose tolerance test; AIR, acute insulin response; NIDDM, non-insulin-dependent diabetes mellitus. jor role in the regulation of insulin secretion. In a rat study conducted in 1990, 24-h infusion of Intralipid (a 10\% triglyceride emulsion) was associated with a decline in beta-cell response to glucose, an effect in direct contrast to the stimulation of insulin secretion that was reported after a shorter-term (3-h) infusion [3]. These results were confirmed in a 1994 in vitro study, which provided evidence that long-term exposure of pancreatic islets to NEFA inhibits glucose-induced insulin secretion and biosynthesis, probably due to a glucose/fatty acid cycle [4]. Recently, fasting plasma NEFA concentrations were shown to predict the development of non-insulin-dependent diabetes mellitus (NIDDM), independent of age, gender, per- 
Table 1. Characteristics of studied subjects

\begin{tabular}{ll}
\hline & Mean (range) \\
\hline$n$ & 12 \\
Age (years) & $36(27-45)$ \\
Gender (male/female) & $5 / 7$ \\
BMI $\left(\mathrm{kg} / \mathrm{m}^{2}\right)$ & $24.7(21-28)$ \\
Body fat $(\%)$ & $29(22-38)$ \\
Fasting plasma glucose $(\mathrm{mmol} / \mathrm{l})$ & $5.0(4.7-5.8)$ \\
Fasting plasma insulin $(\mathrm{pmol} / \mathrm{l})$ & $54(43-61)$ \\
2-h plasma glucose $(\mathrm{mmol} / \mathrm{l})$ & $6.9(6.2-7.5)$ \\
2-h plasma insulin $\left(\mathrm{pmol}^{-1}\right)$ & $318(223-448)$ \\
$\mathrm{M}\left(\mathrm{mg} \cdot \mathrm{kg} \mathrm{FFM}^{-1} \cdot \mathrm{min}^{-1}\right)$ & $6.9(6.3-7.5)$ \\
\hline
\end{tabular}

BMI, Body mass index; $M$, insulin-mediated glucose uptake; FFM, free fat mass

cent body fat, waist/thigh ratio, fasting plasma triglyceride, and insulin-mediated glucose uptake [5]. When acute insulin response (AIR) was added to the model, including gender, percent body fat, and insulin-mediated glucose uptake, fasting plasma NEFA concentrations were no longer predictive of NIDDM [5]. It was thus hypothesized that fasting NEFA concentrations predict the development of NIDDM through effects on both insulin action and secretion.

The above experimental data suggest that longterm exposure of beta cells to high fasting plasma NEFA concentrations may impair insulin secretion. To the best of our knowledge, however, no study has examined the effect of long-term NEFA infusion on glucose-induced insulin secretion in healthy subjects. Therefore, we used a continuous infusion of triglyceride emulsion to investigate the difference in beta-cell response to glucose pulse after short-term (6-h) and long-term (24-h) exposure to high fasting plasma NEFA concentrations.

\section{Subjects and methods}

Subjects. Twelve healthy individuals volunteered for our study (Table 1). These participants were not obese and had normal glucose tolerance defined according to World Health Organization criteria [6]; none had a personal or family history of diabetes. During 3 days prior to undergoing the metabolic investigations and throughout the study periods, all participants consumed a weight-maintaining diet consisting of $50 \%$ carbohydrates, $30 \%$ lipids and $20 \%$ proteins. Insulin-mediated glucose uptake was assessed by a euglycaemic glucose clamp with an insulin infusion rate of $1 \mathrm{mU} / \mathrm{kg}$ per min. None of the participants was taking any drug in the 4 weeks preceding the tests. All individuals gave informed consent prior to participation in the study, which was approved by the ethical committee of our institution.

Experimental design. All studies were performed after a 12-14$\mathrm{h}$ fast and with subjects on bed rest and supine for the duration of testing. An antecubital vein in one arm was cannulated with a 19-gauge catheter for administration of the various agents and kept open by a slow infusion $0.9 \%$ sodium chloride (NaCl). All blood samples were drawn from a contralateral vein through a three-way stopcock to avoid additional venipunctures during the infusion. Three basal samples were obtained before the start of the infusion and the mean of three was considered as the basal value. All participants underwent a $24-\mathrm{h}$ Intralipid $(10 \%$ triglyceride emulsion, Intralipid, Pharmacia, Milan, Italy) infusion at a rate of $0.4 \mathrm{ml} / \mathrm{min}$ and a simultaneous infusion of heparin (a bolus of $200 \mathrm{U}$ followed by $0.2 \mathrm{U} / \mathrm{min}$ per $\mathrm{kg}$ body weight) to raise plasma NEFA concentrations two- to threefold. At baseline, at $6 \mathrm{~h}$, and at the end of the $24 \mathrm{~h}$ period, an intravenous glucose tolerance test (ivGTT; $25 \mathrm{~g} / \mathrm{min}$ ) was administered to assess AIR. Twentyfour hours after the end of the Intralipid infusion, a repeat ivGTT was administered to study the recovery of beta-cell response to glucose. During this study period all subjects stayed in our unit but were not kept in a supine position. After the baseline and the short-term (6-h) test, all the subjects were allowed to eat our standardized diet for healthy subjects. In eight randomly chosen subjects (mean age $35.1 \pm 0.5$ years, gender ratio $5 \mathrm{males} / 3$ females, BMI $24.1 \pm 0.3 \mathrm{~kg} / \mathrm{m}^{2}$, body fat $28.4 \pm 0.5 \%$ ) the same experimental design was repeated along with the infusion of an equal volume of $0.9 \% \mathrm{NaCl}$. Before each ivGTT, changes in respiratory quotient (RQ) (Deltatrac, Datex, Milan, Italy [7]) were also measured for $60 \mathrm{~min}$. Body mass and fat-free mass were determined by bioimpedance [8].

Blood sampling. Blood samples were collected in pre-chilled tubes, which were kept in an ice bath until the end of the study and immediately centrifuged after each experiment. The resultant plasma was stored in a deep freezer for future hormone assay. Plasma glucose concentrations were measured by a glucose oxidase method adapted for use with a Beckman glucose analyzer (Beckman, Fullerton, Calif., USA). Plasma insulin concentration was measured by radioimmunoassay as described elsewhere [9]. Fasting plasma NEFA values were derived using the mean of four different samples drawn at 10min intervals. NEFA concentrations were measured in triplicate on each sample, according to Dole and Meinertz [10]

\section{Statistical analysis}

AIR to glucose was calculated as the incremental area of postglucose insulin levels from 3 to $7 \mathrm{~min}$ [11-12]. Glucose disappearance constant (Conard's $\mathrm{K}$ value or $\mathrm{K}_{\mathrm{g}}$ ) was calculated using the least squares method to determine the slope of correlation between concentration and the time between 10 and $30 \mathrm{~min}$ after glucose bolus [12].

Statistical analyses were performed using SOLO (BMDP, Cork, Republic of Ireland) software package. All values are presented as means $\pm \mathrm{SD}$. Analysis of variance (ANOVA) with repeated measures was used to evaluate the effect of time on the metabolic parameters along with Intralipid infusion. Correlations are Pearson product-moment correlations.

\section{Results}

Intralipid infusion study $(n=12)$. Compared to baseline concentrations, Intralipid infusion caused a three-fold rise in plasma NEFA concentrations, with no difference between 6- and 24-h fasting plasma NEFA concentrations. Fasting plasma glucose concentrations rose progressively throughout the course of the study and were significantly higher at the end 

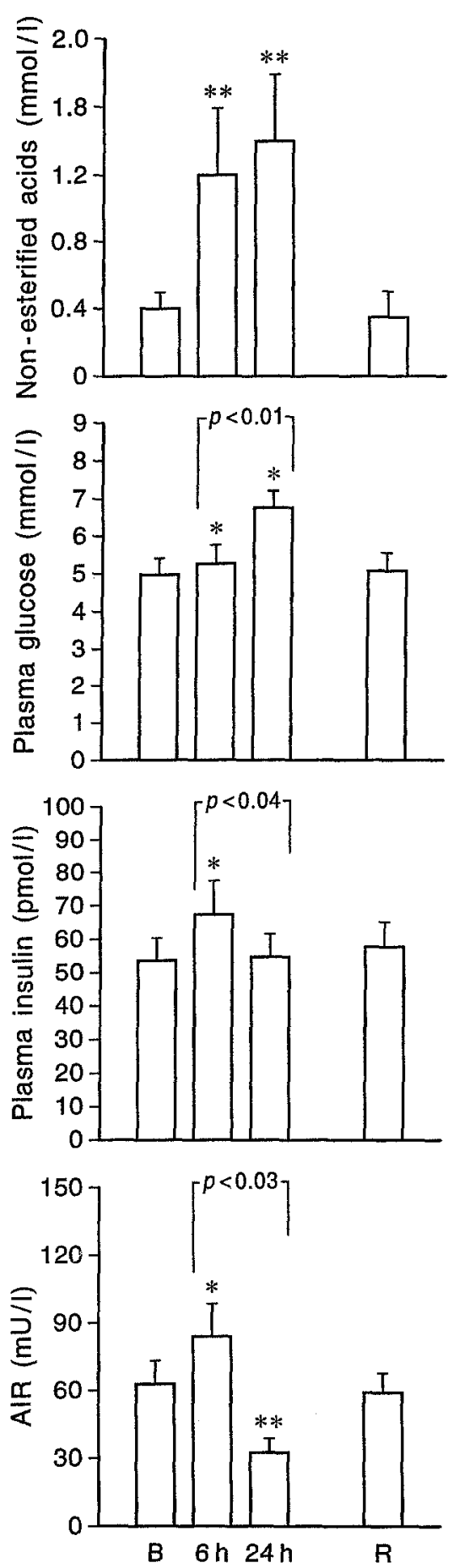

Fig. 1. Fasting plasma NEFA, glucose and insulin concentrations and AIR at the different study times. B, Baseline; $6 \mathrm{~h}$, after 6-h Intralipid infusion; $24 \mathrm{~h}$, after 24 -h Intralipid infusion; $\mathrm{R}$, recovery (after 24-h wash-out period). All results are means $\pm \mathrm{SD} \quad(n=12)$. Statistically significant differences vs baseline were: $* p<0.01, * * p<0.001$

of the Intralipid infusion. Fasting plasma insulin concentrations were significantly higher after $6 \mathrm{~h}$ of infusion, but these concentrations returned to basal values at the end of the infusion. Although AIR was significantly potentiated after $6 \mathrm{~h}$ of infusion, a marked inhibition of AIR occurred with the 24-h infusion.
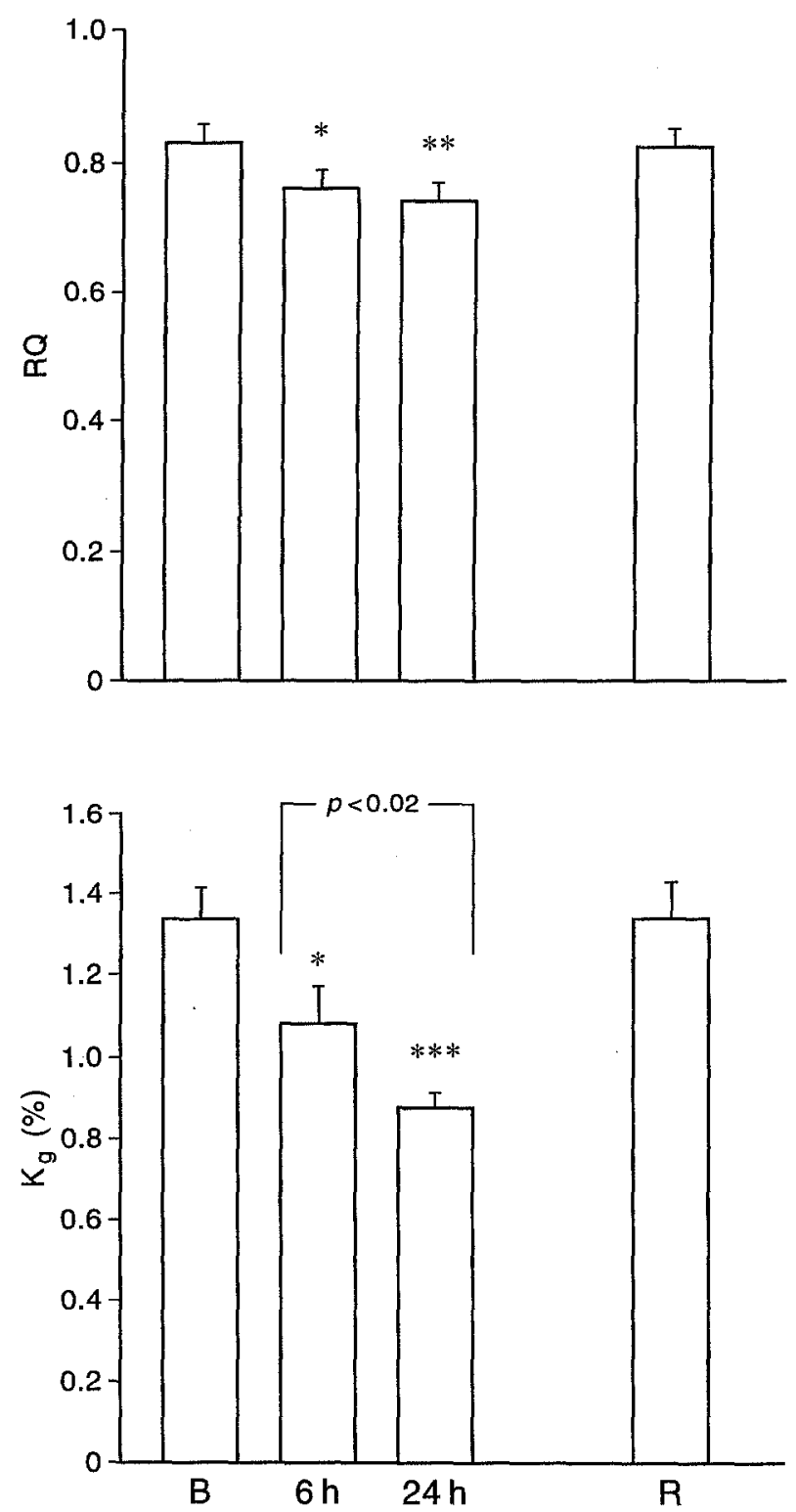

Fig.2. Respiratory quotient (RQ) and glucose disappearance constant $\left(\mathrm{K}_{\mathrm{g}}\right)$ at the different study times. B, Baseline; $6 \mathrm{~h}$, after 6-h Intralipid infusion; $24 \mathrm{~h}$, after 24-h Intralipid infusion; $\mathrm{R}$, recovery (after 24-h wash-out period). All results are means $\pm \mathrm{SD}(n=12)$. Statistically significant differences vs baseline were: $* p<0.04, * * p<0.01, * * * p<0.001$

This latter value was lower than those at baseline and after $6 \mathrm{~h}$ of infusion (Fig. 1).

After the 24-h washout (recovery) period fasting plasma NEFA, glucose and insulin concentrations, and AIR had essentially returned to baseline values (Fig. 1).

Changes in basal $\mathrm{RQ}$ and glucose disappearance constant $\left(\mathrm{K}_{\mathrm{g}}\right)$ throughout the study are shown in Figure 2. RQ was progressively lowered by the Intralipid infusion; i.e., significantly different from basal values after 6 and $24 \mathrm{~h}$; no difference, however, was observed between the 6 - and $24-\mathrm{h}$ values. $\mathrm{K}_{\mathrm{g}}$ was significantly lower than the basal value after the 6 and $24 \mathrm{~h}$ infusion. At the end of the infusion, the strongest inhibi- 

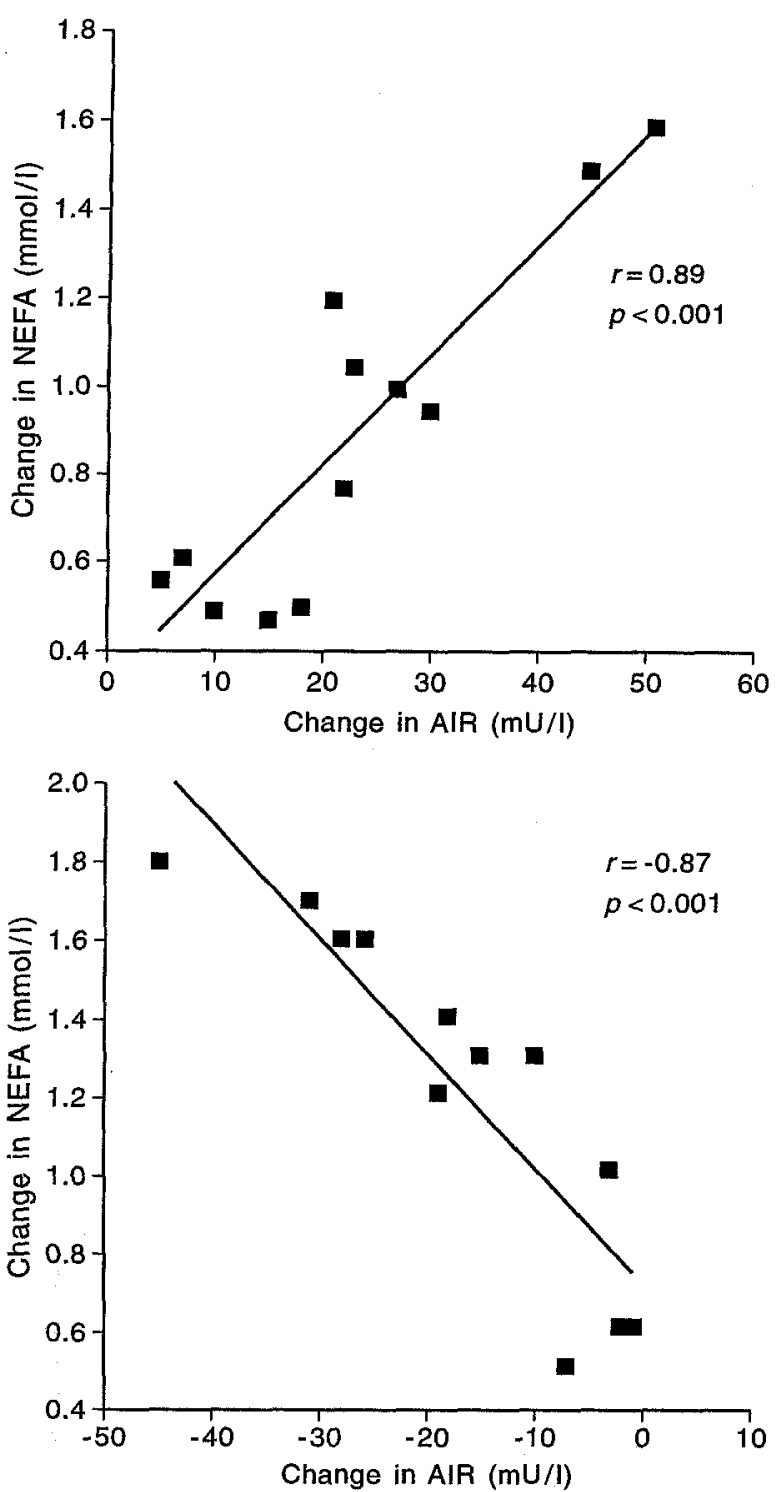

Fig.3. Correlations between changes in fasting plasma (NEFA) and changes AIR after 6-h (top) and 24-h (bottom) Intralipid infusion

tion in $K_{g}$ was found. After the 24-h washout period, $\mathrm{RQ}$ and $\mathrm{K}_{\mathrm{g}}$ had returned to basal values.

After $6 \mathrm{~h}$ of infusion, a positive correlation was found between changes in fasting plasma NEFA concentrations and AIR (Fig. 3). In contrast, at the end of the infusion period, changes in plasma NEFA concentrations and AIR were negatively correlated (Fig. 3).

Control study $(n=8)$. Before starting an infusion of $0.9 \% \mathrm{NaCl}$ solution, fasting plasma NEFA $(0.4 \pm 0.2$ vs $0.4 \pm 0.1 \mathrm{mmol} / \mathrm{l} ; p=\mathrm{NS})$, glucose $(5.1 \pm 0.3$ vs $5.2 \pm 0.2 \mathrm{mmol} / \mathrm{l} ; p=\mathrm{NS})$ and insulin $(56 \pm 12 \mathrm{vs}$ $53 \pm 18 \mathrm{pmol} / \mathrm{l} ; p=\mathrm{NS})$ concentrations, and AIR $(63 \pm 17$ vs $65 \pm 13 \mathrm{mU} / \mathrm{l} ; p=\mathrm{NS})$ were similar to those found before Intralipid infusion. Infusion of the $\mathrm{NaCl}$ solution was not associated with an increase in fasting plasma NEFA, glucose or insulin concen- trations or RQ from baseline values either at the 6-h or 24-h points. Furthermore, AIR remained at baseline after $6 \mathrm{~h}(67 \pm 12 \mathrm{mU} / \mathrm{l} ; p=\mathrm{NS})$ and $24 \mathrm{~h}$ $(61 \pm 15 \mathrm{mU} / \mathrm{l} ; p=\mathrm{NS})$ of $\mathrm{NaCl}$ solution infusion.

\section{Discussion}

Our study demonstrates that short-term (6-h) lipid infusion with a threefold increase in fasting plasma NEFA concentrations stimulates beta-cell response to glucose in healthy volunteers. In contrast, longterm (24-h) lipid infusion was associated with inhibited glucose-induced insulin secretion despite the lack of difference between 6- and 24-h fasting plasma NEFA concentrations.

Thirty years ago, Randle et al. [13-14] proposed that the glucose/fatty acid cycle might cause the alteration in insulin action observed in long-term fasting healthy subjects and in those with NIDDM. Since that time, a growing body of experimental evidence has shown that high plasma NEFA concentrations impair oxidative glucose metabolism and insulin action and cause a secondary impairment in non-oxidative glucose metabolism. The relationship between fatty acid oxidation and glucose metabolism has been documented in skeletal muscle, heart muscle and liver tissue [14]. Despite the fact that fatty acids are also actively taken up and metabolized by pancreatic islets [15], the relationship between plasma NEFA and insulin secretion is still not completely understood.

Numerous studies have shown that acute increases in plasma NEFA concentrations are associated with a stronger beta-cell response to glucose $[1,2]$. In contrast, only few and conflicting data have been reported regarding long-term exposure of beta cells to high plasma NEFA concentrations [16-18].

In order to better emphasize the relationship between NEFA and insulin secretion, Borg [19] incubated pancreatic islets for 7 days in the presence of octanoate, but the results were not conclusive. In 1990, Sako and Grill [3] studied the effects of 48-h lipid infusion on glucose-induced insulin secretion, using an intravenous rather than oral glucose load to stimulate beta-cell response to glucose. In perfused pancreata, hyperlipidaemia, with elevated plasma NEFA concentrations, was associated both with short-term stimulation and long-term inhibition of glucose-induced insulin secretion [3]. Such an effect was present during normo- and hyperglycaemic states. In a 1992 study in diabetic rats, high-fat diets were shown to inhibit glucose-induced insulin secretion, biosynthesis, and oxidation [20]. Zhou and Grill [4] demonstrated that long-term exposure of rat pancreatic islets to high NEFA concentrations inhibits glucose-induced insulin secretion and biosynthesis, probably via activation of the Randle cycle [13]. The inhibitory effect of NEFA was independent of the 
glucose concentration in the incubation medium; this effect was reduced by switching from palmitate to oleate in the medium [13]. Our results are in agreement with those previously reported for animals $[3,20]$ and rat pancreatic islet studies [4].

It is possible that long-term exposure of pancreatic beta cells to high plasma NEFA concentrations might impair glucose-induced insulin secretion through fatty-acid-induced damage of beta-cell plasma membranes. In our study, however, the long-term inhibitory effect of NEFA on glucose-induced insulin secretion was completely reversed by the 24 -h washout period. Further evidence of a specific inhibitory role of high plasma NEFA concentrations also comes from in vitro data showing that the effect of NEFA upon glucose-induced insulin release is rapidly reversed by administration of etomoxir [4], a potent and irreversible carnitine palmitoyl-transferase I inhibitor.

The different effects of plasma NEFA on glucoseinduced insulin secretion after 6 and $24 \mathrm{~h}$ are not surprising. Increased AIR after short-term NEFA infusion may reflect a compensatory reaction on the part of the pancreatic islets to the progressive impairment in glucose handling. On the other hand, it has been postulated in studies with liver tissue that the inhibition of pyruvate dehydrogenase is responsible for the fatty-acid-induced decline in islet glucose oxidation, a process that exhibits a lag time [14].

It should be emphasized that the Intralipid solution used in our study contains a much higher proportion of unsaturated fatty acids than does plasma, with the major fatty acid component being linoleic acid. Opara et al. [21] showed that a 20-min exposure of islet cells to $5 \mathrm{mmol} / \mathrm{l}$ palmitate increased glucose-induced insulin secretion; use of linoleate, however, had the opposite effet. The effect of palmitate - the main plasma fatty acid in glucose-induced insulin secretion - requires future investigation in humans.

In conclusion our study demonstrates that longterm exposure of beta cells to high plasma NEFA concentrations is associated with inhibition of glucose-induced insulin secretion. These data provide a mechanism for further elucidation of the role of elevated NEFA on the development of NIDDM.

Acknowledgement. We are sincerely grateful to Dr. B. V.Howard (Medlantic Research Institute, Washington, DC, USA) who reviewed in depth our results as well as data presentation and discussion.

\section{References}

1. Yamaguchi K, Takoshima S, Masuyama T, Matsuoka A (1978) Effects of electric stress on insulin secretion and glucose metabolism in rats fed with a high fat diet. Endocrinol Jpn 29: 415-419

2. Crespin SR, Greenough WB, Steinberg D (1973) Stimulation of insulin secretion by long-chain free fatty acids. J Clin Invest 53: 1979-1984
3. Sako Y, Grill V (1990) A 48-h lipid infusion in the rat timedependently inhibits glucose-induced insulin secretion and $\beta$-cell oxidation through a process likely coupled to fatty acid oxidation. Endocrinology 127: 1580-1589

4. Zhou YP, Grill V (1994) Long-term exposure of rat pancreatic islets to fatty acids inhibits glucose-induced insulin secretion and biosynthesis through a glucose fatty acid cycle. J Clin Invest 93: 870-876

5. Paolisso G, Tataranni PA, Bogardus C, Howard BV, Ravus$\sin \mathrm{E}(1994)$ High concentration of FFA is a risk factor for the development of NIDDM. Diabetes 43 [Suppl 1] 75A (Abstract)

6. Diabetes mellitus (1985) Report of a WHO study group. World Health Organization 727, pp 9-17

7. Ferrannini E (1990) The theoretical basis of indirect calorimetry: methodological and interpretative problems. $\mathrm{Me}$ tabolism 258: 339-402

8. Segal KR, Van Loan M, Fitzgerald PI, Hodgdon JA, Van Itallie TB (1988) Lean body mass estimation by bioelectrical impedance analysis: a four site cross-validation study. Am J Clin Nutr 47: 7-13

9. Paolisso G, Di Maro G, Pizza G, D'Amore A, Sgambato S, Tesauro P, Varricchio M, D'Onofrio F (1992) Plasma GSH/GSSG affects glucose homeostasis in healthy subjects and non-insulin dependent diabetics. Am J Physiol 263: E435-E440

10. Dole VP, Meinertz H (1960) Microdetermination of longchain fatty acids in plasma and tissues. J Biol Chem 235: 2595-2599

11. Polonsky KS, Rubenstein AH (1986) Current approach to measurements of insulin secretion. Diabetes Metab Rev 2: 315-330

12. Tillil H, Shapiro ET, Miller MA et al. (1988) Dose dependent effect of oral and intravenous glucose on insulin secretion and clearance in normal man. Am J Physiol 17: E349E357

13. Randle PL, Garland PB, Hales CN, Newsholme EA (1963) The glucose fatty acid cycle, its role in insulin sensitivity and the metabolic disturbances in diabetes mellitus. Lancet I. 785-789

14. Randle PJ, Priestman DA, Mistry S, Halsall A (1994) Mechanisms modifying glucose oxidation in diabetes mellitus. Diabetologia 37 [Suppl 2]: S155-S161

15 . Berne $C(1975)$ The oxidation of fatty acids and ketone bodies in mouse pancreatic islets. Biochem J 152: 661-665

16. Goberna R, Tamarit J, Osoris J, Fussgauger R, Tamarit J, Pfeiffer EF (1974) Action of B-hydroxybutirate, acetoacetate and palmitate on the insulin release from the perfused isolated rat pancreas. Horm Metab Res 6: 256-259

17. Malaisse WJ, Malaisse-Lagae F (1968) Stimulation of insulin secretion by non-carbohydrate metabolism. J Lab Clin Med 72: 438-448

18. Malaisse WJ, Lemonnier D, Malaisse-Lagae F, Mondelbaum IM (1969) Secretion of and sensitivity to insulin in obese rats fed a high fat diet. Horm Metab Res 1: 9-12

19. Borg LAH (1981) Effect of octanoate and ketone bodies on the structure and function of isolated pancreatic islets in tissue culture. Acta Endocrinol 96: 505-509

20. Capito K, Hansen SE, Hedeskov CJ, Thomas PJ (1992) Fat induced changes in mouse pancreatic islets, insulin secretion, insulin biosynthesis and glucose metabolism. Acta Diabetol 28: 193-198

21. Opara EC, Garfinkel M, Hubbard VS, Burch WM, Akwari. OE (1994) Effect of fatty acids on insulin release: role of chain length and degree of unsaturation. Am J Physiol 266: E635-E639 